\title{
Search for Large Extra Spatial Dimensions in the Dielectron and Diphoton Channels in $p \bar{p}$ Collisions at $\sqrt{s}=1.96 \mathrm{TeV}$
}

V. M. Abazov,${ }^{36}$ B. Abbott,${ }^{75}$ M. Abolins,${ }^{65}$ B. S. Acharya, ${ }^{29}$ M. Adams,${ }^{51}$ T. Adams, ${ }^{49}$ E. Aguilo, ${ }^{6}$ M. Ahsan, ${ }^{59}$ G. D. Alexeev,${ }^{36}$ G. Alkhazov, ${ }^{40}$ A. Alton, ${ }^{64, *}$ G. Alverson, ${ }^{63}$ G. A. Alves, ${ }^{2}$ M. Anastasoaie,${ }^{35}$ L. S. Ancu, ${ }^{35}$ T. Andeen, ${ }^{53}$ B. Andrieu, ${ }^{17}$ M. S. Anzelc, ${ }^{53}$ M. Aoki,${ }^{50}$ Y. Arnoud,${ }^{14}$ M. Arov, ${ }^{60}$ M. Arthaud,${ }^{18}$ A. Askew, ${ }^{49}$ B. Åsman,${ }^{41}$

A. C. S. Assis Jesus, ${ }^{3}$ O. Atramentov, ${ }^{49}$ C. Avila,${ }^{8}$ F. Badaud,${ }^{13}$ L. Bagby, ${ }^{50}$ B. Baldin,${ }^{50}$ D. V. Bandurin, ${ }^{59}$ P. Banerjee,${ }^{29}$ S. Banerjee, ${ }^{29}$ E. Barberis ${ }^{63}$ A.-F. Barfuss,${ }^{15}$ P. Bargassa,${ }^{80}$ P. Baringer,${ }^{58}$ J. Barreto, ${ }^{2}$ J. F. Bartlett,${ }^{50}$ U. Bassler, ${ }^{18}$ D. Bauer, ${ }^{43}$ S. Beale, ${ }^{6}$ A. Bean, ${ }^{58}$ M. Begalli, ${ }^{3}$ M. Begel, ${ }^{73}$ C. Belanger-Champagne, ${ }^{41}$ L. Bellantoni,${ }^{50}$ A. Bellavance, ${ }^{50}$ J. A. Benitez, ${ }^{65}$ S. B. Beri, ${ }^{27}$ G. Bernardi, ${ }^{17}$ R. Bernhard, ${ }^{23}$ I. Bertram, ${ }^{42}$ M. Besançon, ${ }^{18}$ R. Beuselinck,${ }^{43}$ V. A. Bezzubov, ${ }^{39}$ P. C. Bhat ${ }^{50}$ V. Bhatnagar, ${ }^{27}$ C. Biscarat, ${ }^{20}$ G. Blazey, ${ }^{52}$ F. Blekman, ${ }^{43}$ S. Blessing, ${ }^{49} \mathrm{~K}$. Bloom, ${ }^{67}$ A. Boehnlein, ${ }^{50}$ D. Boline, ${ }^{62}$ T. A. Bolton, ${ }^{59}$ E. E. Boos, ${ }^{38}$ G. Borissov, ${ }^{42}$ T. Bose, ${ }^{77}$ A. Brandt, ${ }^{78}$ R. Brock, ${ }^{65}$ G. Brooijmans, ${ }^{70}$ A. Bross,${ }^{50}$ D. Brown, ${ }^{81}$ X. B. Bu, ${ }^{7}$ N. J. Buchanan, ${ }^{49}$ D. Buchholz, ${ }^{53}$ M. Buehler, ${ }^{81}$ V. Buescher, ${ }^{22}$ V. Bunichev, ${ }^{38}$ S. Burdin, ${ }^{42, \dagger}$ T. H. Burnett, ${ }^{82}$ C. P. Buszello, ${ }^{43}$ J. M. Butler,${ }^{62}$ P. Calfayan, ${ }^{25}$ S. Calvet, ${ }^{16}$ J. Cammin,${ }^{71}$ M. A. Carrasco-Lizarraga, ${ }^{33}$ E. Carrera,${ }^{49}$ W. Carvalho, ${ }^{3}$ B. C. K. Casey,${ }^{50}$ H. Castilla-Valdez, ${ }^{33}$ S. Chakrabarti, ${ }^{18}$ D. Chakraborty, ${ }^{52}$ K. M. Chan,${ }^{55}$ A. Chandra, ${ }^{48}$ E. Cheu, ${ }^{45}$ F. Chevallier, ${ }^{14}$ D. K. Cho, ${ }^{62}$ S. Choi, ${ }^{32}$ B. Choudhary, ${ }^{28}$ L. Christofek, ${ }^{77}$ T. Christoudias, ${ }^{43}$ S. Cihangir, ${ }^{50}$ D. Claes,${ }^{67}$ J. Clutter, ${ }^{58}$ M. Cooke, ${ }^{50}$ W. E. Cooper, ${ }^{50}$ M. Corcoran, ${ }^{80}$ F. Couderc,${ }^{18}$ M.-C. Cousinou, ${ }^{15}$ S. Crépé-Renaudin, ${ }^{14}$ V. Cuplov,${ }^{59}$ D. Cutts,${ }^{77}$ M. Ćwiok,${ }^{30}$ H. da Motta,${ }^{2}$ A. Das, ${ }^{45}$ G. Davies, ${ }^{43}$ K. De,${ }^{78}$ S. J. de Jong, ${ }^{35}$ E. De La Cruz-Burelo, ${ }^{33}$ C. De Oliveira Martins, ${ }^{3}$ K. DeVaughan ${ }^{67}$ F. Déliot,${ }^{18}$ M. Demarteau ${ }^{50}$ R. Demina, ${ }^{71}$ D. Denisov,${ }^{50}$ S. P. Denisov, ${ }^{39}$ S. Desai, ${ }^{50}$ H. T. Diehl, ${ }^{50}$ M. Diesburg, ${ }^{50}$ A. Dominguez, ${ }^{67}$

T. Dorland, ${ }^{82}$ A. Dubey, ${ }^{28}$ L. V. Dudko, ${ }^{38}$ L. Duflot, ${ }^{16}$ S. R. Dugad, ${ }^{29}$ D. Duggan, ${ }^{49}$ A. Duperrin, ${ }^{15}$ J. Dyer, ${ }^{65}$

A. Dyshkant, ${ }^{52}$ M. Eads, ${ }^{67}$ D. Edmunds, ${ }^{65}$ J. Ellison, ${ }^{48}$ V. D. Elvira, ${ }^{50}$ Y. Enari, ${ }^{77}$ S. Eno, ${ }^{61}$ P. Ermolov, ${ }^{38, \dagger}$ H. Evans, ${ }^{54}$ A. Evdokimov, ${ }^{73}$ V. N. Evdokimov, ${ }^{39}$ A. V. Ferapontov, ${ }^{59}$ T. Ferbel,${ }^{71}$ F. Fiedler, ${ }^{24}$ F. Filthaut,${ }^{35}$ W. Fisher, ${ }^{50}$ H. E. Fisk, ${ }^{50}$ M. Fortner, ${ }^{52}$ H. Fox,${ }^{42}$ S. Fu, ${ }^{50}$ S. Fuess,${ }^{50}$ T. Gadfort, ${ }^{70}$ C. F. Galea, ${ }^{35}$ C. Garcia, ${ }^{71}$ A. Garcia-Bellido, ${ }^{71}$ V. Gavrilov, ${ }^{37}$ P. Gay, ${ }^{13}$ W. Geist, ${ }^{19}$ W. Geng, ${ }^{15,65}$ C. E. Gerber, ${ }^{51}$ Y. Gershtein, ${ }^{49,}$ D. Gillberg, ${ }^{6}$ G. Ginther, ${ }^{71}$ B. Gómez, ${ }^{8}$ A. Goussiou, ${ }^{82}$

P. D. Grannis, ${ }^{72}$ H. Greenlee,${ }^{50}$ Z. D. Greenwood ${ }^{60}$ E. M. Gregores,${ }^{4}$ G. Grenier, ${ }^{20}$ Ph. Gris, ${ }^{13}$ J.-F. Grivaz, ${ }^{16}$

A. Grohsjean, ${ }^{25}$ S. Grünendahl, ${ }^{50}$ M. W. Grünewald, ${ }^{30}$ F. Guo, ${ }^{72}$ J. Guo, ${ }^{72}$ G. Gutierrez, ${ }^{50}$ P. Gutierrez, ${ }^{75}$ A. Haas ${ }^{70}$

N. J. Hadley, ${ }^{61}$ P. Haefner, ${ }^{25}$ S. Hagopian, ${ }^{49}$ J. Haley ${ }^{68}$ I. Hall ${ }^{65}$ R. E. Hall, ${ }^{47}$ L. Han, ${ }^{7}$ K. Harder ${ }^{44}$ A. Harel, ${ }^{71}$ J. M. Hauptman, ${ }^{57}$ J. Hays,${ }^{43}$ T. Hebbeker, ${ }^{21}$ D. Hedin, ${ }^{52}$ J. G. Hegeman ${ }^{34}$ A. P. Heinson, ${ }^{48}$ U. Heintz, ${ }^{62}$ C. Hensel, ${ }^{22, \S}$ K. Herner, ${ }^{72}$ G. Hesketh, ${ }^{63}$ M. D. Hildreth, ${ }^{55}$ R. Hirosky, ${ }^{81}$ J. D. Hobbs, ${ }^{72}$ B. Hoeneisen, ${ }^{12}$ M. Hohlfeld, ${ }^{22}$ S. Hossain, ${ }^{75}$ P. Houben, ${ }^{34}$ Y. Hu, ${ }^{72}$ Z. Hubacek, ${ }^{10}$ V. Hynek, ${ }^{9}$ I. Iashvili, ${ }^{69}$ R. Illingworth, ${ }^{50}$ A. S. Ito, ${ }^{50}$ S. Jabeen, ${ }^{62}$ M. Jaffré, ${ }^{16}$ S. Jain, ${ }^{75}$ K. Jakobs,${ }^{23}$ C. Jarvis,${ }^{61}$ R. Jesik,${ }^{43}$ K. Johns,${ }^{45}$ C. Johnson, ${ }^{70}$ M. Johnson,${ }^{50}$ D. Johnston, ${ }^{67}$ A. Jonckheere, ${ }^{50}$ P. Jonsson, ${ }^{43}$ A. Juste, ${ }^{50}$ E. Kajfasz,${ }^{15}$ D. Karmanov, ${ }^{38}$ P. A. Kasper, ${ }^{50}$ I. Katsanos, ${ }^{70}$ D. Kau, ${ }^{49}$ V. Kaushik, ${ }^{78}$ R. Kehoe,${ }^{79}$ S. Kermiche,${ }^{15}$

N. Khalatyan, ${ }^{50}$ A. Khanov, ${ }^{76}$ A. Kharchilava, ${ }^{69}$ Y. M. Kharzheev, ${ }^{36}$ D. Khatidze, ${ }^{70}$ T. J. Kim, ${ }^{31}$ M. H. Kirby ${ }^{53}$ M. Kirsch ${ }^{21}$ B. Klima, ${ }^{50}$ J. M. Kohli, ${ }^{27}$ J.-P. Konrath, ${ }^{23}$ A. V. Kozelov, ${ }^{39}$ J. Kraus, ${ }^{65}$ T. Kuhl, ${ }^{24}$ A. Kumar ${ }^{69}$ A. Kupco, ${ }^{11}$ T. Kurča, ${ }^{20}$ V. A. Kuzmin, ${ }^{38}$ J. Kvita, ${ }^{9}$ F. Lacroix,${ }^{13}$ D. Lam,${ }^{55}$ S. Lammers,${ }^{70}$ G. Landsberg, ${ }^{77}$ P. Lebrun, ${ }^{20}$ W. M. Lee,${ }^{50}$ A. Leflat,${ }^{38}$ J. Lellouch, ${ }^{17}$ J. Li, ${ }^{78,8 \S}$ L. Li, ${ }^{48}$ Q. Z. Li ${ }^{50}$ S. M. Lietti, ${ }^{5}$ J. K. Lim, ${ }^{31}$ J. G. R. Lima, ${ }^{52}$ D. Lincoln, ${ }^{50}$ J. Linnemann, ${ }^{65}$ V. V. Lipaev, ${ }^{39}$ R. Lipton, ${ }^{50}$ Y. Liu, ${ }^{7}$ Z. Liu, ${ }^{6}$ A. Lobodenko, ${ }^{40}$ M. Lokajicek, ${ }^{11}$ P. Love, ${ }^{42}$ H. J. Lubatti, ${ }^{82}$ R. Luna-Garcia, ${ }^{33, \|}$ A. L. Lyon, ${ }^{50}$ A. K. A. Maciel, ${ }^{2}$ D. Mackin,${ }^{80}$ R. J. Madaras ${ }^{46}$ P. Mättig, ${ }^{26}$ C. Magass, ${ }^{21}$

A. Magerkurth, ${ }^{64}$ P. K. Mal, ${ }^{82}$ H. B. Malbouisson, ${ }^{3}$ S. Malik, ${ }^{67}$ V. L. Malyshev, ${ }^{36}$ Y. Maravin,${ }^{59}$ B. Martin, ${ }^{14}$

R. McCarthy, ${ }^{72}$ M. M. Meijer, ${ }^{35}$ A. Melnitchouk, ${ }^{66}$ L. Mendoza, ${ }^{8}$ P. G. Mercadante, ${ }^{5}$ M. Merkin, ${ }^{38}$ K. W. Merritt, ${ }^{50}$ A. Meyer, ${ }^{21}$ J. Meyer, ${ }^{22,8}$ J. Mitrevski, ${ }^{70}$ R. K. Mommsen, ${ }^{44}$ N. K. Mondal,${ }^{29}$ R. W. Moore, ${ }^{6}$ T. Moulik, ${ }^{58}$ G. S. Muanza, ${ }^{15}$ M. Mulhearn, ${ }^{70}$ O. Mundal, ${ }^{22}$ L. Mundim,${ }^{3}$ E. Nagy, ${ }^{15}$ M. Naimuddin, ${ }^{50}$ M. Narain, ${ }^{77}$ N. A. Naumann ${ }^{35}$ H. A. Neal, ${ }^{64}$ J. P. Negret, ${ }^{8}$ P. Neustroev,${ }^{40}$ H. Nilsen, ${ }^{23}$ H. Nogima, ${ }^{3}$ S. F. Novaes, ${ }^{5}$ T. Nunnemann, ${ }^{25}$ V. O'Dell, ${ }^{50}$ D. C. O'Neil, ${ }^{6}$ G. Obrant, ${ }^{40}$ C. Ochando, ${ }^{16}$ D. Onoprienko, ${ }^{59}$ N. Oshima, ${ }^{50}$ N. Osman, ${ }^{43}$ J. Osta, ${ }^{55}$ R. Otec,${ }^{10}$ G. J. Otero y Garzón, ${ }^{50}$ M. Owen,${ }^{44}$ P. Padley,${ }^{80}$ M. Pangilinan,${ }^{77}$ N. Parashar,${ }^{56}$ S.-J. Park, ${ }^{22,8}$ S. K. Park,${ }^{31}$ J. Parsons,${ }^{70}$ R. Partridge,${ }^{77}$ N. Parua,${ }^{54}$ A. Patwa, ${ }^{73}$ G. Pawloski, ${ }^{80}$ B. Penning, ${ }^{23}$ M. Perfilov,${ }^{38}$ K. Peters, ${ }^{44}$ Y. Peters, ${ }^{26}$ P. Pétroff,,${ }^{16}$ M. Petteni,${ }^{43}$ R. Piegaia, ${ }^{1}$ J. Piper ${ }^{65}$ M.-A. Pleier, ${ }^{22}$ P. L. M. Podesta-Lerma, ${ }^{33, \pi}$ V. M. Podstavkov, ${ }^{50}$ Y. Pogorelov, ${ }^{55}$ M.-E. Pol, ${ }^{2}$ P. Polozov, ${ }^{37}$ B. G. Pope, ${ }^{65}$ A. V. Popov,${ }^{39}$ C. Potter, ${ }^{6}$ W. L. Prado da Silva, ${ }^{3}$ H. B. Prosper, ${ }^{49}$ S. Protopopescu, ${ }^{73}$ J. Qian,${ }^{64}$ A. Quadt, ${ }^{22, \S}$ B. Quinn, ${ }^{66}$ A. Rakitine, ${ }^{42}$ M. S. Rangel, ${ }^{2}$ K. Ranjan, ${ }^{28}$ P. N. Ratoff, ${ }^{42}$ P. Renkel, ${ }^{79}$ P. Rich, ${ }^{44}$ M. Rijssenbeek,${ }^{72}$ 
I. Ripp-Baudot, ${ }^{19}$ F. Rizatdinova, ${ }^{76}$ S. Robinson, ${ }^{43}$ R. F. Rodrigues, ${ }^{3}$ M. Rominsky, ${ }^{75}$ C. Royon, ${ }^{18}$ P. Rubinov, ${ }^{50}$ R. Ruchti, ${ }^{55}$ G. Safronov, ${ }^{37}$ G. Sajot,${ }^{14}$ A. Sánchez-Hernández,${ }^{33}$ M. P. Sanders, ${ }^{17}$ B. Sanghi, ${ }^{50}$ G. Savage, ${ }^{50}$ L. Sawyer ${ }^{60}$ T. Scanlon, ${ }^{43}$ D. Schaile, ${ }^{25}$ R. D. Schamberger ${ }^{72}$ Y. Scheglov, ${ }^{40}$ H. Schellman, ${ }^{53}$ T. Schliephake, ${ }^{26}$ S. Schlobohm, ${ }^{82}$ C. Schwanenberger ${ }^{44}$ A. Schwartzman, ${ }^{68}$ R. Schwienhorst ${ }^{65}$ J. Sekaric ${ }^{49}$ H. Severini ${ }^{75}$ E. Shabalina,${ }^{51}$ M. Shamim, ${ }^{59}$ V. Shary, ${ }^{18}$ A. A. Shchukin, ${ }^{39}$ R. K. Shivpuri, ${ }^{28}$ V. Siccardi, ${ }^{19}$ V. Simak, ${ }^{10}$ V. Sirotenko, ${ }^{50}$ P. Skubic, ${ }^{75}$ P. Slattery, ${ }^{71}$

D. Smirnov, ${ }^{55}$ G. R. Snow, ${ }^{67}$ J. Snow, ${ }^{74}$ S. Snyder, ${ }^{73}$ S. Söldner-Rembold, ${ }^{44}$ L. Sonnenschein, ${ }^{17}$ A. Sopczak, ${ }^{42}$

M. Sosebee, ${ }^{78}$ K. Soustruznik, ${ }^{9}$ B. Spurlock ${ }^{78}$ J. Stark, ${ }^{14}$ V. Stolin, ${ }^{37}$ D. A. Stoyanova, ${ }^{39}$ J. Strandberg, ${ }^{64}$ S. Strandberg, ${ }^{41}$ M. A. Strang, ${ }^{69}$ E. Strauss, ${ }^{72}$ M. Strauss, ${ }^{75}$ R. Ströhmer, ${ }^{25}$ D. Strom,${ }^{53}$ L. Stutte,${ }^{50}$ S. Sumowidagdo, ${ }^{49}$ P. Svoisky, ${ }^{35}$ A. Sznajder, ${ }^{3}$ A. Tanasijczuk, ${ }^{1}$ W. Taylor, ${ }^{6}$ B. Tiller ${ }^{25}$ F. Tissandier, ${ }^{13}$ M. Titov, ${ }^{18}$ V. V. Tokmenin,${ }^{36}$ I. Torchiani, ${ }^{23}$ D. Tsybychev, ${ }^{72}$ B. Tuchming, ${ }^{18}$ C. Tully,${ }^{68}$ P. M. Tuts,${ }^{70}$ R. Unalan, ${ }^{65}$ L. Uvarov, ${ }^{40}$ S. Uvarov, ${ }^{40}$ S. Uzunyan, ${ }^{52}$ B. Vachon, ${ }^{6}$ P. J. van den Berg, ${ }^{34}$ R. Van Kooten,${ }^{54}$ W. M. van Leeuwen, ${ }^{34}$ N. Varelas, ${ }^{51}$ E. W. Varnes, ${ }^{45}$ I. A. Vasilyev,${ }^{39}$ P. Verdier,${ }^{20}$ L. S. Vertogradov, ${ }^{36}$ M. Verzocchi ${ }^{50}$ D. Vilanova, ${ }^{18}$ F. Villeneuve-Seguier,${ }^{43}$ P. Vint, ${ }^{43}$ P. Vokac, ${ }^{10}$ M. Voutilainen, ${ }^{67, * *}$ R. Wagner, ${ }^{68}$ H. D. Wahl,${ }^{49}$ M.H. L. S. Wang, ${ }^{50}$ J. Warchol,${ }^{55}$ G. Watts,${ }^{82}$ M. Wayne, ${ }^{55}$ G. Weber, ${ }^{24, \dagger \dagger}$ M. Weber, ${ }^{50, \dagger \dagger}$ L. Welty-Rieger, ${ }^{54}$ A. Wenger, ${ }^{23,+1}$ N. Wermes, ${ }^{22}$ M. Wetstein, ${ }^{61}$ A. White, ${ }^{78}$ D. Wicke, ${ }^{26}$ M. Williams,${ }^{42}$ G. W. Wilson, ${ }^{58}$ S. J. Wimpenny, ${ }^{48}$ M. Wobisch, ${ }^{60}$ D. R. Wood ${ }^{63}$ T. R. Wyatt, ${ }^{44}$ Y. Xie, ${ }^{77}$ C. Xu, ${ }^{64}$ S. Yacoob,${ }^{53}$ R. Yamada,${ }^{50}$ W.-C. Yang, ${ }^{44}$ T. Yasuda ${ }^{50}$ Y. A. Yatsunenko, ${ }^{36}$ H. Yin, ${ }^{7}$ K. Yip, ${ }^{73}$ H. D. Yoo, ${ }^{77}$ S. W. Youn, ${ }^{53}$ J. Yu, ${ }^{78}$ C. Zeitnitz, ${ }^{26}$ S. Zelitch,${ }^{81}$ T. Zhao, ${ }^{82}$ B. Zhou, ${ }^{64}$ J. Zhu, ${ }^{72}$ M. Zielinski, ${ }^{71}$ D. Zieminska, ${ }^{54}$ A. Zieminski, ${ }^{54, \S \S}$ L. Zivkovic, ${ }^{70}$ V. Zutshi, ${ }^{52}$ and E. G. Zverev ${ }^{38}$

(D0 Collaboration)

\author{
${ }^{1}$ Universidad de Buenos Aires, Buenos Aires, Argentina \\ ${ }^{2}$ LAFEX, Centro Brasileiro de Pesquisas Físicas, Rio de Janeiro, Brazil \\ ${ }^{3}$ Universidade do Estado do Rio de Janeiro, Rio de Janeiro, Brazil \\ ${ }^{4}$ Universidade Federal do ABC, Santo André, Brazil \\ ${ }^{5}$ Instituto de Física Teórica, Universidade Estadual Paulista, São Paulo, Brazil \\ ${ }^{6}$ University of Alberta, Edmonton, Alberta, Canada, \\ Simon Fraser University, Burnaby, British Columbia, Canada, \\ York University, Toronto, Ontario, Canada, \\ and McGill University, Montreal, Quebec, Canada \\ ${ }^{7}$ University of Science and Technology of China, Hefei, People's Republic of China \\ ${ }^{8}$ Universidad de los Andes, Bogotá, Colombia \\ ${ }^{9}$ Center for Particle Physics, Charles University, Prague, Czech Republic \\ ${ }^{10}$ Czech Technical University, Prague, Czech Republic \\ ${ }^{11}$ Center for Particle Physics, Institute of Physics, Academy of Sciences of the Czech Republic, Prague, Czech Republic \\ ${ }^{12}$ Universidad San Francisco de Quito, Quito, Ecuador \\ ${ }^{13}$ LPC, Université Blaise Pascal, CNRS/IN2P3, Clermont, France \\ ${ }^{14}$ LPSC, Université Joseph Fourier Grenoble 1, CNRS/IN2P3, Institut National Polytechnique de Grenoble, Grenoble, France \\ ${ }^{15}$ CPPM, Aix-Marseille Université, CNRS/IN2P3, Marseille, France \\ ${ }^{16}$ LAL, Université Paris-Sud, IN2P3/CNRS, Orsay, France \\ ${ }^{17}$ LPNHE, IN2P3/CNRS, Universités Paris VI and VII, Paris, France \\ ${ }^{18}$ CEA, Irfu, SPP, Saclay, France \\ ${ }^{19}$ IPHC, Université Louis Pasteur, CNRS/IN2P3, Strasbourg, France \\ ${ }^{20} I P N L$, Université Lyon 1, CNRS/IN2P3, Villeurbanne, France and Université de Lyon, Lyon, France \\ ${ }^{21}$ III. Physikalisches Institut A, RWTH Aachen University, Aachen, Germany \\ ${ }^{22}$ Physikalisches Institut, Universität Bonn, Bonn, Germany \\ ${ }^{23}$ Physikalisches Institut, Universität Freiburg, Freiburg, Germany \\ ${ }^{24}$ Institut für Physik, Universität Mainz, Mainz, Germany \\ ${ }^{25}$ Ludwig-Maximilians-Universität München, München, Germany \\ ${ }^{26}$ Fachbereich Physik, University of Wuppertal, Wuppertal, Germany \\ ${ }^{27}$ Panjab University, Chandigarh, India \\ ${ }^{28}$ Delhi University, Delhi, India \\ ${ }^{29}$ Tata Institute of Fundamental Research, Mumbai, India \\ ${ }^{30}$ University College Dublin, Dublin, Ireland \\ ${ }^{31}$ Korea Detector Laboratory, Korea University, Seoul, Korea \\ ${ }^{32}$ SungKyunKwan University, Suwon, Korea \\ ${ }^{33}$ CINVESTAV, Mexico City, Mexico
}


${ }^{34}$ FOM-Institute NIKHEF and University of Amsterdam/NIKHEF, Amsterdam, The Netherlands

${ }^{35}$ Radboud University Nijmegen/NIKHEF, Nijmegen, The Netherlands

${ }^{36}$ Joint Institute for Nuclear Research, Dubna, Russia

${ }^{37}$ Institute for Theoretical and Experimental Physics, Moscow, Russia

${ }^{38}$ Moscow State University, Moscow, Russia

${ }^{39}$ Institute for High Energy Physics, Protvino, Russia

${ }^{40}$ Petersburg Nuclear Physics Institute, St. Petersburg, Russia

${ }^{41}$ Lund University, Lund, Sweden, Royal Institute of Technology and Stockholm University, Stockholm, Sweden, and Uppsala University, Uppsala, Sweden

${ }^{42}$ Lancaster University, Lancaster, United Kingdom

${ }^{43}$ Imperial College, London, United Kingdom

${ }^{44}$ University of Manchester, Manchester, United Kingdom

${ }^{45}$ University of Arizona, Tucson, Arizona 85721, USA

${ }^{46}$ Lawrence Berkeley National Laboratory and University of California, Berkeley, California 94720, USA

${ }^{47}$ California State University, Fresno, California 93740, USA

${ }^{48}$ University of California, Riverside, California 92521, USA

${ }^{49}$ Florida State University, Tallahassee, Florida 32306, USA

${ }^{50}$ Fermi National Accelerator Laboratory, Batavia, Illinois 60510, USA

${ }^{51}$ University of Illinois at Chicago, Chicago, Illinois 60607, USA

${ }^{52}$ Northern Illinois University, DeKalb, Illinois 60115, USA

${ }^{53}$ Northwestern University, Evanston, Illinois 60208, USA

${ }^{54}$ Indiana University, Bloomington, Indiana 47405, USA

${ }^{55}$ University of Notre Dame, Notre Dame, Indiana 46556, USA

${ }^{56}$ Purdue University Calumet, Hammond, Indiana 46323, USA

${ }^{57}$ Iowa State University, Ames, Iowa 50011, USA

${ }^{58}$ University of Kansas, Lawrence, Kansas 66045, USA

${ }^{59}$ Kansas State University, Manhattan, Kansas 66506, USA

${ }^{60}$ Louisiana Tech University, Ruston, Louisiana 71272, USA

${ }^{61}$ University of Maryland, College Park, Maryland 20742, USA

${ }^{62}$ Boston University, Boston, Massachusetts 02215, USA

${ }^{63}$ Northeastern University, Boston, Massachusetts 02115, USA

${ }^{64}$ University of Michigan, Ann Arbor, Michigan 48109, USA

${ }^{65}$ Michigan State University, East Lansing, Michigan 48824, USA

${ }^{66}$ University of Mississippi, University, Mississippi 38677, USA

${ }^{67}$ University of Nebraska, Lincoln, Nebraska 68588, USA

${ }^{68}$ Princeton University, Princeton, New Jersey 08544, USA

${ }^{69}$ State University of New York, Buffalo, New York 14260, USA

${ }^{70}$ Columbia University, New York, New York 10027, USA

${ }^{71}$ University of Rochester, Rochester, New York 14627, USA

${ }^{72}$ State University of New York, Stony Brook, New York 11794, USA

${ }^{73}$ Brookhaven National Laboratory, Upton, New York 11973, USA

${ }^{74}$ Langston University, Langston, Oklahoma 73050, USA

${ }^{75}$ University of Oklahoma, Norman, Oklahoma 73019, USA

${ }^{76}$ Oklahoma State University, Stillwater, Oklahoma 74078, USA

${ }^{77}$ Brown University, Providence, Rhode Island 02912, USA

${ }^{78}$ University of Texas, Arlington, Texas 76019, USA

${ }^{79}$ Southern Methodist University, Dallas, Texas 75275, USA

${ }^{80}$ Rice University, Houston, Texas 77005, USA

${ }^{81}$ University of Virginia, Charlottesville, Virginia 22901, USA

${ }^{82}$ University of Washington, Seattle, Washington 98195, USA

(Received 18 September 2008; published 6 February 2009)

We report on a search for large extra spatial dimensions in the dielectron and diphoton channels using a data sample of $1.05 \mathrm{fb}^{-1}$ of $p \bar{p}$ collisions at a center-of-mass energy of $1.96 \mathrm{TeV}$ collected by the D0 detector at the Fermilab Tevatron Collider. The invariant mass spectrum of the data agrees well with the prediction of the standard model. We find the most restrictive 95\% C.L. lower limits on the effective Planck scale between 2.1 and $1.3 \mathrm{TeV}$ for 2 to 7 extra dimensions. 
Within the standard model (SM) the mass of Higgs boson is unstable against radiative corrections. The fact that the mass is not of the order of the GUT or Planck scales at $10^{16}$ or $10^{19} \mathrm{GeV}$ but rather $\mathcal{O}\left(10^{2} \mathrm{GeV}\right)$ is commonly referred to as the "hierarchy problem." One way to circumvent the neeed for such fine-tuning in the Higgs boson mass is by extending the dimentionality of the space, as in the large extra dimension model (LED) proposed by Arkani-Hamed, Dimopoulos, and Dvali (ADD) [1], which posits that the fields of the standard model are pinned to a $(3+1)$-dimensional membrane, while gravity propagates in $n_{d}$ additional compactified spatial dimensions. Gauss' Law gives the relation between the fundamental Planck scale $M_{D}$, the observed Planck scale $M_{\mathrm{Pl}}$, and the size of the extra dimensions $R$ : $\left[M_{\mathrm{Pl}}\right]^{2} \approx R^{n_{d}}\left[M_{D}\right]^{n_{d}+2}$. If $R$ is large compared to the Planck length $\simeq 1.6 \times 10^{-33} \mathrm{~cm}$, $M_{D}$ can be as low as $\mathcal{O}(1 \mathrm{TeV})$, thus avoiding the hierarchy problem and making gravity strong at the $\mathrm{TeV}$ scale. Extra spatial dimensions will manifest themselves by the presence of a series of graviton states, known as a "KaluzaKlein tower", $\left(G_{\mathrm{KK}}\right)$. At colliders, large extra dimensions can be probed by searching for the effect of $G_{\mathrm{KK}}$ on fermion or boson pair production [2].

Extra dimension amplitudes will result in enhancement of the cross sections above the SM values, especially at high energies. The LED cross section, which consists of $\mathrm{SM}$, interference, and direct gravity terms, can be parametrized by a single variable $\eta_{G}=F / M_{s}^{4}$ where $M_{s}$ is the effective Planck scale, the ultraviolet cutoff of the sum over Kaluza-Klein states in virtual graviton exchange. The exact relationship between $M_{s}$ and $M_{D}$ depends on the exact quantum gravity scenario although they are of the same order of magnitude. The dimensionless parameter $F$ to leading order (LO) and the subleading $n_{d}$ dependence is given by

$$
F=1,
$$

(GRW [3]),

$$
F= \begin{cases}\ln \left(M_{s}^{2} / \hat{s}\right) & \text { for } n_{d}=2, \\ \frac{2}{n_{d}-2} & \text { for } n_{d}>2\end{cases}
$$

(HLZ [4]), where $\hat{s}$ is the center-of-mass energy of the partonic subprocess.

In this Letter, we present a search for LED performed in events containing an $e^{+} e^{-}$or $\gamma \gamma$ pair with $1.05 \mathrm{fb}^{-1}$ of $p \bar{p}$ collider data collected with the upgraded D0 detector [5] between October 2002 and February 2006. With $127 \mathrm{pb}^{-1}$ of data, D0 has published limits on $M_{s}$ ranging from 1.0 to $1.4 \mathrm{TeV}$ for $n_{d}=7-2$ in the combined dielectron and diphoton channels [6]. Less restrictive limits in the same final state are also reported in [7]. D0 has also published limits in the dimuon channel with $246 \mathrm{pb}^{-1}$ of data [8]. The efficiency and resolution for high energy electromagnetic (EM) objects at D0 are superior to those for muons and so a search for LED in combined $e^{+} e^{-}$and $\gamma \gamma(\mathrm{di}-$
EM) final states is superior to the dimuon channel. D0 and CDF have also published limits on $M_{D}$ in the monophoton and monophoton plus monojet final states, respectively [9].

Events are collected using triggers requiring the presence of at least one EM calorimeter shower with the transverse momentum with respect to the beam axis, $p_{T}$, greater than $15 \mathrm{GeV}$. From these data we select $e^{+} e^{-}$and $\gamma \gamma$ events using criteria that do not distinguish photons from electrons. We require events with two EM showers with $p_{T}>25 \mathrm{GeV}$. Showers are labeled CC (EC) if they are reconstructed in the central calorimeter (end cap calorimeters) with $|\eta|<1.1(1.5<|\eta|<2.4)$, where pseudorapidity $\eta=-\ln [\tan (\theta / 2)]$ and $\theta$ is the polar angle measured with respect to the proton beam direction. To reduce multijet background, we require at least one shower to be in the CC, so that selected events are either CC-CC (both showers in the CC) or CC-EC (one shower in the CC and the other in the EC). Each EM shower is required to be isolated, with less than $7 \%$ of the cluster energy in an annular cone $0.2<\Delta \mathcal{R}<0.4$ about the shower centroid, where $\Delta \mathcal{R}=\sqrt{(\Delta \eta)^{2}+(\Delta \phi)^{2}}$ and $\phi$ is the azimuthal angle. We also demand the scalar sum of the $p_{T}$ of all tracks in the cone $0.05<\Delta \mathcal{R}<0.4$ be less than $2 \mathrm{GeV}$. Finally, we demand the EM shower profile be consistent with that of an electron or photon using a $\chi^{2}$ test and that $97 \%$ of the shower energy be contained in the EM calorimeter.

The efficiencies for the electron and the photon selection criteria are determined from the same data set used for the event selection. We estimate separately the efficiencies for the $\chi^{2}$ requirement on the EM shower shape, the isolation requirements based on $\Delta \mathcal{R}$, and for all calorimeter-based high- $p_{T}$ triggers relevant to this analysis. In order to estimate the different efficiencies, we select a sample of di-EM events satisfying very loose EM identification requirements with invariant mass within $\pm 40 \mathrm{GeV}$ around $Z$ boson mass. For each of these di-EM candidate events we estimate the efficiency as a function of $\eta$ versus $p_{T}$ using the tag and probe method [10]. This efficiency is then applied to Monte Carlo simulation samples.

The irreducible background to the LED signal is from $\mathrm{SM} e^{+} e^{-}$and $\gamma \gamma$ production, while instrumental background arises from multijet and $\gamma+$ jet events with jets misidentified as EM objects. To model the invariant mass distribution of the physics backgrounds, we use the PYTHIA [11] event generator using the CTEQ6L1 parton distribution functions [12], followed by a GEANT-based [13] detector simulation and reconstruction with the same algorithms as applied to data. The next-to-leading order (NLO) effect for both $e^{+} e^{-}$and $\gamma \gamma$ is taken into account by multiplying the leading order (LO) cross section by a mass independent $k$-factor of 1.34 [14].

We generate the LED signal for $2 \leq n_{d} \leq 7$ and 33 different values of $M_{s}$ using a parton level generator [15]. Following [4], we assume $\operatorname{Br}\left(G_{\mathrm{KK}} \rightarrow \gamma \gamma\right) / \operatorname{Br}\left(G_{\mathrm{KK}} \rightarrow e^{+} e^{-}\right)=$ 2. In order to model the effects of detector resolution and 
TABLE I. Number of events observed and expected from SM processes in different mass windows for CC-CC and CC-EC events. The individual contributions to the total SM expectation from multijet, $e^{+} e^{-}$and $\gamma \gamma$ are also shown separately.

CC-CC

CC-EC

\begin{tabular}{|c|c|c|c|c|c|c|c|c|c|c|c|}
\hline $\begin{array}{l}\text { Mass } \\
(\mathrm{GeV})\end{array}$ & $\begin{array}{l}\text { Data } \\
N\end{array}$ & $\begin{array}{l}\text { Total Background } \\
\qquad N_{b} \pm N_{b}^{\text {sys }} \\
\end{array}$ & $\begin{array}{c}\text { Multijet(MJ) } \\
N_{\mathrm{MJ}} \pm N_{\mathrm{MJ}}^{\text {sys }} \\
\end{array}$ & $\begin{array}{l}e^{+} e^{-} \\
N_{e^{+} e^{-}}\end{array}$ & $\begin{array}{r}\gamma \gamma \\
N_{\gamma \gamma} \\
\end{array}$ & $\begin{array}{c}\text { Data } \\
N\end{array}$ & $\begin{array}{l}\text { Total Background } \\
\qquad N_{b} \pm N_{b}^{\text {sys }} \\
\end{array}$ & $\begin{array}{l}\text { Multijet(MJ) } \\
N_{\mathrm{MJ}} \pm N_{\mathrm{MJ}}^{\mathrm{sys}}\end{array}$ & & $\begin{array}{l}e^{+} e^{-} \\
N_{e^{+} e^{-}} \\
\end{array}$ & $\begin{array}{c}\gamma \gamma \\
N_{\gamma \gamma} \\
\end{array}$ \\
\hline 240-290 & 61 & $67 \pm 8$ & $22 \pm 3.1$ & 30 & 15 & 144 & $171 \pm 34$ & 115 & 34 & 34 & 21 \\
\hline 290-340 & 30 & $28 \pm 4$ & $7 \pm 1$ & 14 & 7 & 52 & $55 \pm 11$ & 35 & 11 & 12 & 8 \\
\hline 340-400 & 21 & $15 \pm 2$ & $3 \pm 1$ & 7 & 5 & 21 & $23 \pm 5$ & 12 & 4 & 7 & 4 \\
\hline $400-500$ & 9 & $9 \pm 1$ & $1.4 \pm 0.3$ & 5 & 3 & 12 & $9 \pm 2$ & 4 & 2 & 3.3 & 1.2 \\
\hline 500-600 & 1 & $3.6 \pm 1.2$ & $0.14 \pm 0.09$ & 2.4 & 1.1 & 2 & $1.5 \pm 0.4$ & 0.6 & 0.2 & 0.73 & 0.18 \\
\hline 600-1000 & 2 & $1.3 \pm 0.1$ & $0.11 \pm 0.06$ & 0.67 & 0.53 & 0 & $0.35 \pm 0.07$ & 0.03 & 0.04 & 0.24 & 0.08 \\
\hline
\end{tabular}

initial state radiation (ISR), we generate LED + SM and SM-only events separately to obtain the parton level distributions of the di-EM invariant mass versus the cosine of the scattering angle in the center-of-mass frame of the two EM candidates $\left(\left|\cos \theta^{*}\right|\right)$ for each value of $M_{s}$ and $n_{d}$ considered. The ratio of the LED + SM and SM distributions are obtained for all values of $M_{s}$ and $n_{d}$. Standard model events generated with the detailed GEANT-based Monte Carlo simulation are weighted by this ratio to model the effect of an LED signal. We reweight the shape of the SM to simulate the LED signal, keeping the overall normalization as in the pure SM case. By normalizing to the $Z$ boson production cross section (NNLO), where the signal contribution is negligible, we reduce the fractional uncertainty on the product of the efficiency and integrated luminosity.

To estimate the normalization of the multijet background, we fit the di-EM invariant mass distribution of the selected data events with a linear combination of the physics and instrumental background distributions. The shape of the invariant mass distribution for the instrumental background is estimated from data events with EM energy clusters that fail the $\chi^{2}$ requirement for the shower profile. This fit is performed in the mass range $60-140 \mathrm{GeV}$ where we expect no contribution from LED. We obtain separate fits for CC-CC and CC-EC events. From the fits we determine the fraction $f_{\mathrm{MJ}}$ of the multijet contribution to the total background in the mass range $60-140 \mathrm{GeV}$ to be $f_{\mathrm{MJ}}=0.22 \pm 0.03$ in CC-CC events and $f_{\mathrm{MJ}}=0.24 \pm$ 0.07 in CC-EC events. We extrapolate the total background using the fitted value of $f_{\mathrm{MJ}}$ to determine the expected number of background events with invariant mass above $140 \mathrm{GeV}$ in both the CC-CC and CC-EC configurations. Table I shows the numbers of events in different mass ranges for CC-CC and CC-EC where we would expect the LED signal to appear. The number of events is consistent with the number of expected events from the SM expectation. Figure 1(a) shows the invariant mass distribution for CC-CC events and Fig. 1(b) for CC-EC events. The distributions of $\left|\cos \theta^{*}\right|$ are shown in Fig. 2 for CC-CC and CC-EC both for data and the background model. We find that the total background distribution for the invariant mass and $\left|\cos \theta^{*}\right|$ is consistent with the data within statistical and systematic uncertainties.
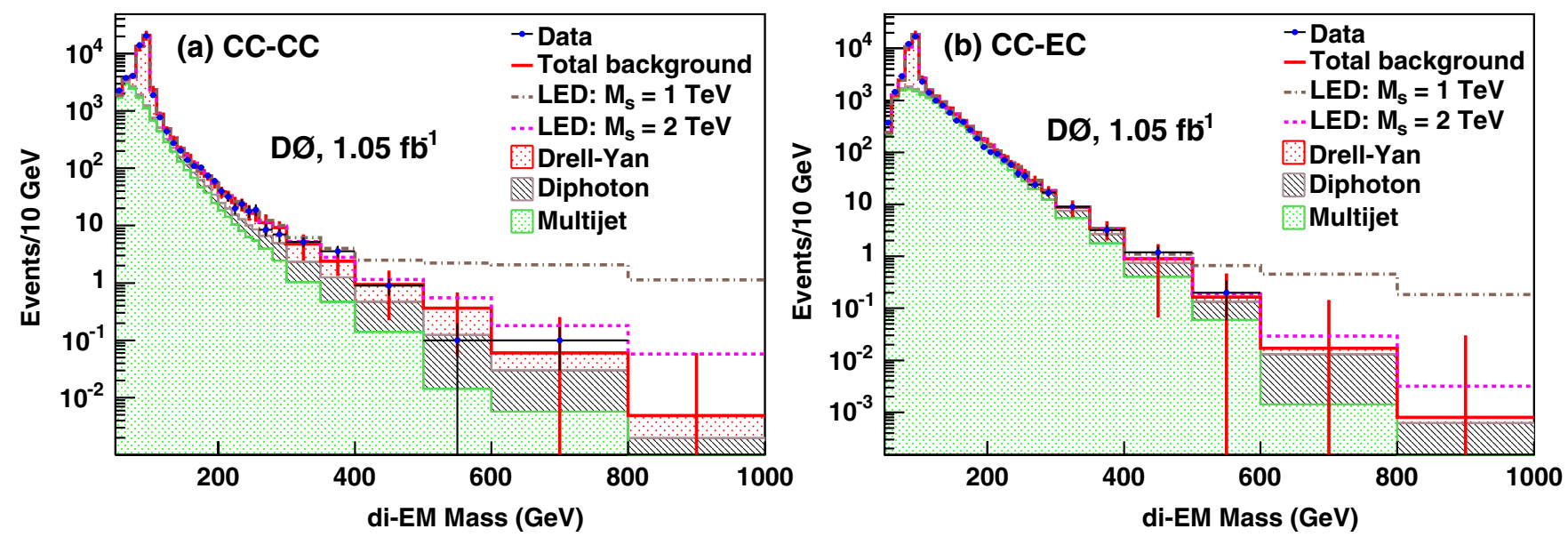

FIG. 1 (color online). The di-EM invariant mass distributions for CC-CC (a) and CC-EC (b) events. The data are shown by points with error bars, the filled histograms represent the Drell-Yan, diphoton, and multijet backgrounds, and the solid line represents the total background. The broken lines show the invariant mass distributions for two different values of $M_{s}$ for $n_{d}=4$. The error bars for the total background include both statistical and systematic uncertainties. 

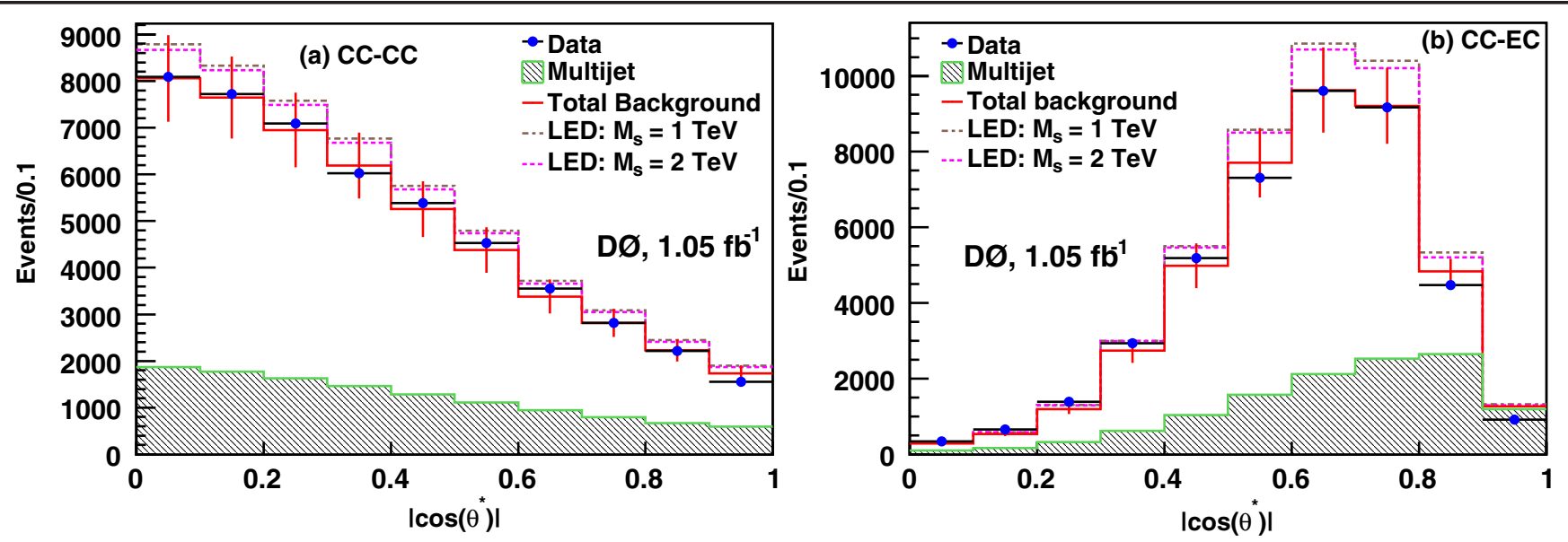

FIG. 2 (color online). The distributions of the center-of-mass scattering angle $\cos \theta^{*}$ of the two final state EM candidates in CC-CC (a) and CC-EC (b) events. The data are shown by points with error bars, the filled histogram represent the multijet background, and the solid line represents the total background. The broken lines show the $\cos \theta^{*}$ distributions for two different values of $M_{s}$ for $n_{d}=4$. The error bars for the total background include both statistical and systematic uncertainties.

Most of the systematic uncertainties on the background model are dependent on the invariant mass. The dominant uncertainty arises from the efficiency of the $\chi^{2}$ cut on the shower profile used to estimate multijet background (13\% of the background itself in CC-CC and 30\% in CC-EC). The systematic on the LED modeling is dominated by uncertainties on the choice of parton distribution functions [(1-19)\% in CC-CC and (1.5-12)\% in CC-EC]. All the other signal uncertainties are correlated to SM background uncertainties due to the technique used to generate our LED signal. Table II summarizes the dominant background and signal uncertainties taken into account in calculating the limit on $M_{s}$. The NLO $k$-factor uncertainty refers to the uncertainty due to choice of PDF, renormalization and factorization scale.

TABLE II. Systematic uncertainties (in \%) on the predicted numbers of signal and background events considered in calculating the limit on $M_{s}$.

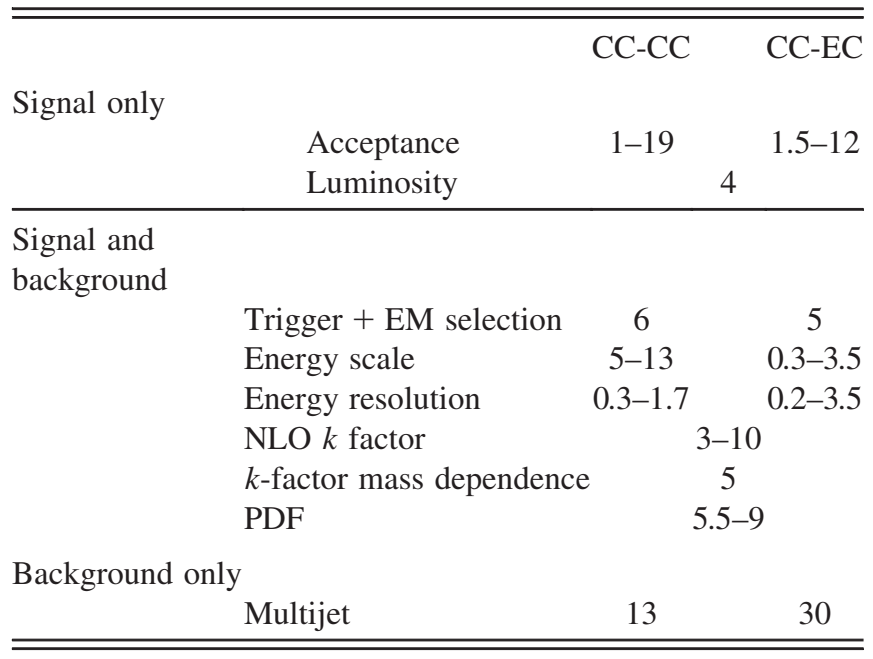

The two-dimensional distribution of the invariant mass and $\left|\cos \theta^{*}\right|$ for the observed dielectron and diphoton events is compared with the corresponding distributions expected from SM physics and instrumental background, and the LED signal for $M_{s}$ ranging from $1 \mathrm{TeV}$ to $3 \mathrm{TeV}$ for a given $n_{d}$. The posterior probability density $P\left(M_{s} \mid\right.$ Data $)$ given the number of observed events in the $k$ th mass bin and $l$ th $\cos \theta^{*}$ bin, $N_{\mathrm{obs}}^{k, l}$, is then computed using a Gaussian prior for the SM plus multijet background. Evidence of large extra dimensions with a given $M_{s}$ will appear as a peak in $P\left(M_{s} \mid\right.$ Data) distribution. In the absence of signal we proceed to estimate the lower limit on $M_{s}$ using the semifrequentist CLs method [16], which is based on computation of a $\log$ likelihood ratio. Both the expected and observed limits on $M_{s}$ at the $95 \%$ C.L. are calculated. Systematic uncertainties in the signal and background distributions are taken into account in this calculation, with their correlations properly included. The distribution of the ratio of the observed (expected) upper limit at the 95\% C.L. limit to the predicted cross section as a function of $M_{s}$ is used to extract the observed (expected) limit on $M_{s}$ for $n_{d}=7$ to $n_{d}=2$.

For the $n_{d}$ independent GRW formalism, we calculate the observed(expected) limit on $M_{s}$ of 1.62(1.66) TeV. We obtain the observed limits on $M_{s}$ at the $95 \%$ C.L. in the HLZ formalism (subleading, $n_{d}$ dependent) ranging from 1.29 to $2.09 \mathrm{TeV}$ for $n_{d}=7$ to $n_{d}=2$. Both the observed

TABLE III. Observed and expected lower limits at the $95 \%$ C.L. on the effective Planck scale, $M_{s}$, in TeV.

\begin{tabular}{lllllllll}
\hline \hline & GRW & \multicolumn{1}{c}{ HLZ } & & & & & \\
\hline & & $n_{d}$ & 2 & 3 & 4 & 5 & 6 & 7 \\
Obs. & 1.62 & & 2.09 & 1.94 & 1.62 & 1.46 & 1.36 & 1.29 \\
Exp. & 1.66 & & 2.16 & 2.01 & 1.66 & 1.49 & 1.38 & 1.31 \\
\hline \hline
\end{tabular}




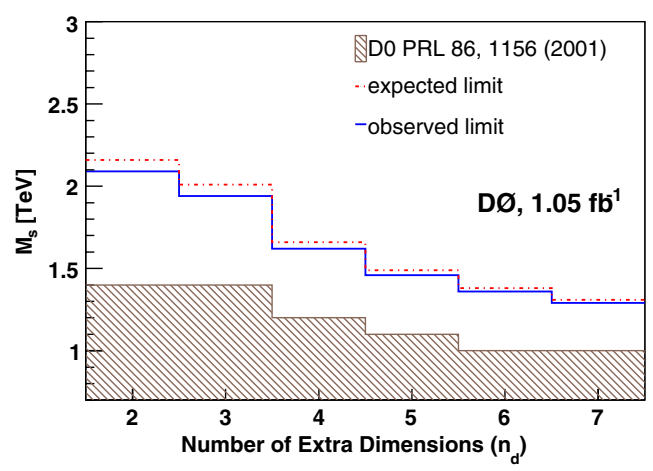

FIG. 3 (color online). Observed and expected limits on the effective Planck scale, $M_{s}$, in the di-EM channel along with previously published limits in di-EM channel.

and expected limits on $M_{s}$, for different formalisms and for six different $n_{d}$ are summarized in Table III. The observed and expected limits on $M_{s}$ for a given number of extra dimensions are found to be similar. The present limits are a significant improvement over the published limit [6]. Figure 3 summarizes the observed and expected limits on $M_{s}$ along with the previously published limits on $M_{s}$ in the di-EM channel.

In summary, we have performed a dedicated search for large extra spatial dimensions by looking for effects of virtual Kaluza-Klein graviton in the dielectron and diphoton channels using $1.05 \mathrm{fb}^{-1}$ of data collected by D0 detector. We see no evidence of excess over the standard model prediction and set limits at 95\% C.L. on the effective Planck scale at 2.09(1.29) $\mathrm{TeV}$ for 2(7) extra dimensions. These are presently the most restrictive limits on the effective Planck scale from searches for large extra dimensions.

We thank the staffs at Fermilab and collaborating institutions, and acknowledge support from the DOE and NSF (USA); CEA and CNRS/IN2P3 (France); FASI, Rosatom and RFBR (Russia); CNPq, FAPERJ, FAPESP and FUNDUNESP (Brazil); DAE and DST (India); Colciencias (Colombia); CONACyT (Mexico); KRF and KOSEF (Korea); CONICET and UBACyT (Argentina); FOM (The Netherlands); STFC (United Kingdom); MSMT and GACR (Czech Republic); CRC Program, CFI, NSERC and WestGrid Project (Canada); BMBF and DFG (Germany); SFI (Ireland); The Swedish Research Council (Sweden); CAS and CNSF (China); and the Alexander von Humboldt Foundation (Germany).

*Visitor from Augustana College, Sioux Falls, SD, USA. ${ }^{\dagger}$ Visitor from The University of Liverpool, Liverpool, United Kingdom.
${ }^{\star}$ Visitor from Rutgers University, Piscataway, NJ, USA.

${ }^{\S}$ Visitor from II. Physikalisches Institut, Georg-AugustUniversity, Göttingen, Germany.

"Visitor from Centro de Investigacion en ComputacionIPN, Mexico City, Mexico.

${ }^{\text {TV} V i s i t o r ~ f r o m ~ E C F M, ~ U n i v e r s i d a d ~ A u t o n o m a ~ d e ~ S i n a l o a, ~}$ Culiacán, Mexico.

**Visitor from Helsinki Institute of Physics, Helsinki, Finland.

${ }^{\dagger}$ Visitor from Universität Bern, Bern, Switzerland.

$\$$ Visitor from Universität Zürich, Zürich, Switzerland.

$\S \S$ Deceased.

[1] N. Arkani-Hamed, S. Dimopoulos, and G. Dvali, Phys. Lett. B 429, 263 (1998); I. Antoniadis, N. Arkani-Hamed, S. Dimopoulos, and G. Dvali, Phys. Lett. B 436, 257 (1998); N. Arkani-Hamed, S. Dimopoulos, and G. Dvali, Phys. Rev. D 59, 086004 (1999); N. Arkani-Hamed, S. Dimopoulos, and J. March-Russell, Phys. Rev. D 63, 064020 (2001).

[2] J. L. Hewett, Phys. Rev. Lett. 82, 4765 (1999); K. Cheung, Phys. Lett. B 460, 383 (1999); K. Cheung and G. Landsberg, Phys. Rev. D 62, 076003 (2000); K. Cheung, Phys. Rev. D 61, 015005 (1999); O. J.P. Eboli et al., Phys. Rev. D 61, 094007 (2000).

[3] G. Giudice, R. Rattazzi, and J. Wells, Nucl. Phys. B544, 3 (1999), and revised version arXiv:hep-ph/9811291.

[4] T. Han, J. Lykken, and R. Zhang, Phys. Rev. D 59, 105006 (1999), and revised version arXiv:hep-ph/9811350.

[5] V.M. Abazov et al. (D0 Collaboration), Nucl. Instrum. Methods Phys. Res., Sect. A 565, 463 (2006).

[6] B. Abbott et al. (D0 Collaboration), Phys. Rev. Lett. 86, 1156 (2001).

[7] D. Gerdes et al., Phys. Rev. D 73, 112008 (2006).

[8] V. M. Abazov et al. (D0 Collaboration), Phys. Rev. Lett. 95, 161602 (2005).

[9] V. M. Abazov et al. (D0 Collaboration), Phys. Rev. Lett. 101, 011601 (2008); T. Aaltonen et al. (CDF Collaboration), Phys. Rev. Lett. 101, 181602 (2008).

[10] V. M. Abazov et al. (D0 Collaboration), Phys. Rev. D 76, 012003 (2007).

[11] T. Sjöstrand, S. Mrenna, and P. Skands, J. High Energy Phys. 05 (2006) 026; we used version 6.323.

[12] J. Pumplin et al., J. High Energy Phys. 07 (2002) 012; D. Stump et al., J. High Energy Phys. 10 (2003) 046.

[13] R. Brun and F. Carminati, CERN Program Library Long Writeup W5013, 1993 (unpublished).

[14] P. Mathews, V. Ravindran, K. Sridhar, and W. L. van Neerven, Nucl. Phys. B713, 333 (2005); R. Hamberg, W. L. van Neerven, and T. Matsuura, Nucl. Phys. B359, 343 (1991); B644, 403(E) (2002).

[15] K. Cheung and G. Landsberg, Phys. Rev. D 62, 076003 (2000).

[16] T. Junk, Nucl. Instrum. Methods Phys. Res., Sect. A 434, 435 (1999); A. Read, CERN Report No. 2000-005 (2000); W. Fisher, FERMILAB-TM-2386-E (2007). 